\title{
Transformational Effect of COVID Pandemic on Postgraduate Programme in Oral and Maxillofacial Surgery in India-A Trainee Perspective
}

\author{
Ankita chugh $^{1} \cdot$ Gaurav Jain $^{2}$ (D) Shubham Gaur ${ }^{1} \cdot$ P. G. Gigi ${ }^{1} \cdot$ Pravin Kumar $^{1}$ • \\ Surjit Singh ${ }^{3}$
}

Received: 2 March 2021 / Accepted: 19 April 2021/Published online: 4 May 2021

(C) The Association of Oral and Maxillofacial Surgeons of India 2021

\begin{abstract}
Background and purpose The purpose of the survey was to evaluate transformation in mode of teaching during the COVID pandemic on a postgraduate program in oral and maxillofacial surgery in India.

Methods A standardised e-questionnaire was created on Google Forms ${ }^{\mathrm{TM}}$ and was shared using emails and WhatsApp ${ }^{\mathrm{TM}}$. A total of 103 postgraduate students of oral and maxillofacial surgery from different universities participated in this cross-sectional survey. The collected data were analysed using bivariate and multivariate analysis.

Results A total of $95.1 \%$ of postgraduates in the survey believed that their overall regular speciality work was deprived during first 5 months of COVID. 90.3\% participants accepted that webinar/online teaching program become more beneficial for their academics. But $78.1 \%$ participants think that because of overburdening of these academic sessions, they have reduced the enthusiasm in attending them.

Conclusion This survey highlighted the intense negative impacts of this pandemic on the postgraduate program from the eyes of the trainees themselves. This triggers us to hasten this process of medical education transformation in a way to cope with any such calamity with minimal consequences.
\end{abstract}

Gaurav Jain

dr.gauravjainn@gmail.com

1 Department of Dentistry, All India Institute of Medical Sciences, Jodhpur, Rajasthan, India

2 Department of Dentistry, All India Institute of Medical Sciences, Deoghar, Jharkhand, India

3 Department of Pharmacology, All India Institute of Medical Sciences, Jodhpur, Rajasthan, India

\section{Introduction}

Coronavirus disease 2019 (COVID-19) is one of its kind which started from Wuhan city, Hubei Province, China in December 2019 as a pneumonia of unknown origin but in no time engulfed the entire globe [1]. World Health Organization (WHO) had announced it as pandemic on March 12, 2020. Since then to minimise and slow its spread, different government and administration agencies enforced various norms like travel restrictions, curfews, mandatory mask, social distancing and public places closures. Education sector was invariably forced for complete closure because of various reasons, like education institute, were having large subset of population with high density, finding it difficult to manage social distancing norms among children and young, and most importantly, it was presumed that closure of educational institutions will have minimal financial and social impacts.

Health workers working in the vicinity of virus lodgements areas with aerosol generation procedures were obviously considered high-risk professionals. Unlike other education sectors, a large number of healthcare workers were to be required for fighting with pandemic, so training of new trainees was also a utmost important task with worldwide lockdowns. So an online teaching mode was initiated, advocated and promoted worldwide especially in healthcare education institutes. This no doubt has widened the ultimate horizon of education system which is thought to now may stay forever [2].

$\mathrm{We}$, oral and maxillofacial surgeons both at nation and worldwide, have also caught up with this new model of education. The flood of knowledge dissemination globally via webinars in these past 10 months have further close knit the maxillofacial fraternity [2]. But could dentistry and especially postgraduate programme in oral and 
maxillofacial surgery which is basically a practical and procedure-based learning speciality doing enough justice to its naive learners in this COVID era is a thought to ponder. This cross-sectional study across the nation was done to evaluate the postgraduate students perspective on the effect of COVID 19 on their academic curriculum and training.

\section{Material and Method}

This was a simple survey-based cross-sectional study designed using electronic questionnaire which was created based on the result of previously conducted small focus group discussion and in-depth interview carried out on a number of selected students and lecturers. The questionnaire was formulated by a group of 3 subject experts consisting of total 32 multiple choice questions divided into 3 groups that is (1) changes in clinical activities, (2) trend in academic activities and (3) examination pattern during pandemic period. The validity of questions was evaluated by an intradepartmental pilot study and further refinement of questionnaire was done. After obtaining ethical clearance from institutional ethics committee, the questionnaire was made on Google Forms ${ }^{\mathrm{TM}}$ along with the consent form for participation in the survey. Then the questionnaire was published online; the link for survey was shared with the students through WhatsApp ${ }^{\mathrm{TM}}$ and Email. The collection of data was done through survey for the period of 45 days from 21 September 2020 to 5 November 2020. After that, all the collected data were put together in Microsoft Excel spreadsheet, then being exported to SPSS $^{\mathrm{TM}}$ ver. 21 for further analysis.

\section{Results}

A total of 103 postgraduate students enrolled during the duration of survey participated in the study. Out of them, $23 \%(n=24)$ were first year residents, $36 \%(n=37)$ were second year and $41 \%(n=42)$ were third year residents. Out of these respondents, $8.7 \%$ percent believes that clinical work in their department has been started to its preCOVID level, $30.1 \%, 35.9 \%$ and $25.2 \%$ believe reinstitution of work is $75 \%, \% 0 \%$ and less than $25 \%$ compared to pre-COVID times.

\section{Discussion}

COVID 2019 has been landmark in revolutionizing our lives both personally and professionally. However for good and hopefully to persist forever it has reinstated the significance of stringent infection control practices and universal precautions in our clinical practice. But this fear during last few months that dental fraternity including oral and maxillofacial surgeons are among highest risk of acquiring and disseminating COVID infection has been devastating mentally, physically and psychologically. Patient care and control of this pandemic was the primary and ultimate motto. Disruption of medical education has happened worldwide as a consequence to it. [3] To assess these effects among oral and maxillofacial trainees, a survey was conducted during the months of September to November. The survey encompassed questions pertaining to phase of complete lockdown of services as well as the phase of restart of clinical services. There was a variation in pattern and timing of reinstitution of clinical work load as the information from survey conveys.

Medical sector or hospitals with their teams were the ones which had worked round the clock during this era for COVID care. However all non-COVID-related procedures and elective procedures were postponed and neglected. Most outpatient clinics had shifted to telemedicine mode. Social distancing restricted the numbers of patients, even when clinics were started physically. Even today by 1 st week of February, it is unsure to say if complete elective array of procedures has started at most of the secondary and tertiary healthcare centres. This has drastically affected the postgraduate curriculum.

$95.1 \%$ of postgraduates in the survey believed that their overall regular speciality work was deprived during first 5 months of COVID (Table 1). 99\% accepted minor surgeries performed independently by them had decreased (Table 1). Out of them, $33.1 \%$ believed there was greater than $80 \%$ reduction in minor surgery cases while $19.4 \%$ percent felt reduction was in tune of 50\% (Table 2). Major elective surgeries had reduced by $>80 \%$, according to $42.7 \%$ respondents (Table 1). Trauma surgeries which are considered the most common surgery in maxillofacial setting are not life threatening hence were considered as semi elective procedures. Thus their number had also declined as reported by $89.3 \%$ of respondents (Table 1 ).

With the restriction in motility of human kind, trauma cases had itself reduced in number but simultaneously during COVID times, closed reduction was preferred over Open reduction and Internal fixation (ORIF) so this also led to decrease in trauma surgery. This study also reported similar pattern with $70.9 \%$ favouring closed reduction in initial months of COVID wherever possible (Table 1). But now pendulum has again swung towards regular preference for ORIF may be because of the thinking that the disease will stay for long. By the end of October, $89.3 \%$ admitted that clinical work had reinstated in their department (graph 1). $35.9 \%$ felt that $50 \%$ resumption of pre-COVID clinical load has been there, but $25 \%$ still felt that actual clinical work now is not even $25 \%$ of regular work (Graph 1). 
Table 1 Response of participant for single choice question

Questions

\section{Response}

Yes No

$(\%)$

1. Do you feel you have been deprived of clinical exposure to your regular specialty work in last 5 months?

$95.1 \quad 4.9$

2. Has the number of minor surgeries performed by postgraduates decreased?

$99 \quad 1$

3 . Is the number of trauma cases that were operated also decreased ?

$89.3 \quad 10.7$

4. Has clinical work been reinstituted at your centre?

5. Do you get COVID testing done by RT-PCR for all OPD-based minor surgery procedures ?

6. When the scare about the disease was high initially Was there any change in treatment choice in trauma patient (Closed v/s 70.9 Open)?

7. Now people have realized that this disease is here to stay yet still the choice of treatment is same or changed from Close to 70.9 Open?

8. What precautions you are taking during any operative procedure (if COVID status Known negative) ?
A. N-95 Mask
$95.1 \quad 4.9$
B. Face shield
$94.1 \quad 5.9$
C. Surgical Gown
$83.4 \quad 16.6$
D. PPE Kit

9. What precautions are mandatory during any operative procedure (if COVID status unknown)?
A. $\mathrm{N}-95$ mask
B. Face shield
C. Surgical Gown
D. PPE Kit

10. Do you use evacuators, external suction devices beyond what were used in Pre-Covid era ?

11. Do you think webinars have broadened academic content for postgraduates by giving them the privilege to hear to national level speakers?

12. Do you think the enthusiasm in attending these webinars is same as it was in the beginning

13. Do you think there has been an overdose of the webinars ?

14. Do you think these long duration webinars are unable to maintain the attention span of participants?

15. Faculty have taken lectures/talks at different forums at pan State/ INDIA level. Do you think faculty should take teaching/ lectures for their students regularly as part of postgraduate curriculum?

16. Do you think the types of online academic activities witnessed during this pandemic can replace conventional method of postgraduate teaching?

17. Do you think these online activities are able to replicate seminar room experience?

A. Inadequate Two-way communication

B. Less interactive

C. Technical glitches

D. Face-to-face encounter maintains the decorum and sanctity of teaching

E. No accountability or check on participation/or even if students are listening or paying attention

18. Should the course be extended for an additional period?

$56.3 \quad 43.7$

19. Is online examination pattern satisfactory to assess a candidate?

65

35

20. Do you think this pandemic (COVID -19) had affected your post-graduate training as a whole (academic and clinical)?
Mandatory Rt-PCR before minor surgery was reported to be performed by only $37.9 \%$ of respondents (Table 1 ).

In COVID times, medical technology had a mega surge in India. Suddenly, the market was flooded with various types of personal protective equipments (PPE), aerosol reducing equipment and mechanisms. Ninety-nine respondents believed that difficulty level associated while working in complete PPE was more than 6 out of 10 (Table 2). Extra oral evacuators or high-vacuum external suction devices became a norm for practice at many places, but it reported to be used by $46.6 \%$ of our respondents (Table 1). This could be because oral and maxillofacial surgery in comparison with other specialities of dentistry 
Table 2 Participant (\%) distribution of response for rating-based questions having rating (0-100\%)

\begin{tabular}{|c|c|c|c|c|c|c|c|c|c|c|}
\hline \multirow[t]{2}{*}{ Question } & \multicolumn{10}{|c|}{ Percentage scale for response } \\
\hline & $10 \%$ & $20 \%$ & $30 \%$ & $40 \%$ & $50 \%$ & $60 \%$ & $70 \%$ & $80 \%$ & $90 \%$ & $100 \%$ \\
\hline $\begin{array}{l}\text { 1. Do you think your exposure of clinical or academics has suffered } \\
\text { to an extent that it has affected your required gain of training }\end{array}$ & 0.97 & 0.97 & 0.97 & 3.88 & 6.80 & 12.62 & 10.68 & 18.45 & 17.48 & 27.18 \\
\hline $\begin{array}{l}\text { 2. How much number of cases operated by postgraduates reduced } \\
\text { after pandemic as compared to pre-COVID era? }\end{array}$ & 5.83 & 4.85 & 8.74 & 5.83 & 19.42 & 11.65 & 10.68 & 16.50 & 11.65 & 4.85 \\
\hline $\begin{array}{l}\text { 3. Has the number of elective Major surgeries performed in the } \\
\text { department also decreased? }\end{array}$ & 8.74 & 3.88 & 5.83 & 9.71 & 10.68 & 8.74 & 9.71 & 16.50 & 13.59 & 12.62 \\
\hline 4. How difficult is it to work with full PPE Kit? & 0.00 & 1.94 & 0.00 & 1.94 & 5.83 & 7.77 & 13.59 & 26.21 & 23.30 & 19.42 \\
\hline
\end{tabular}

Graph 1 Potential up to which clinical work started

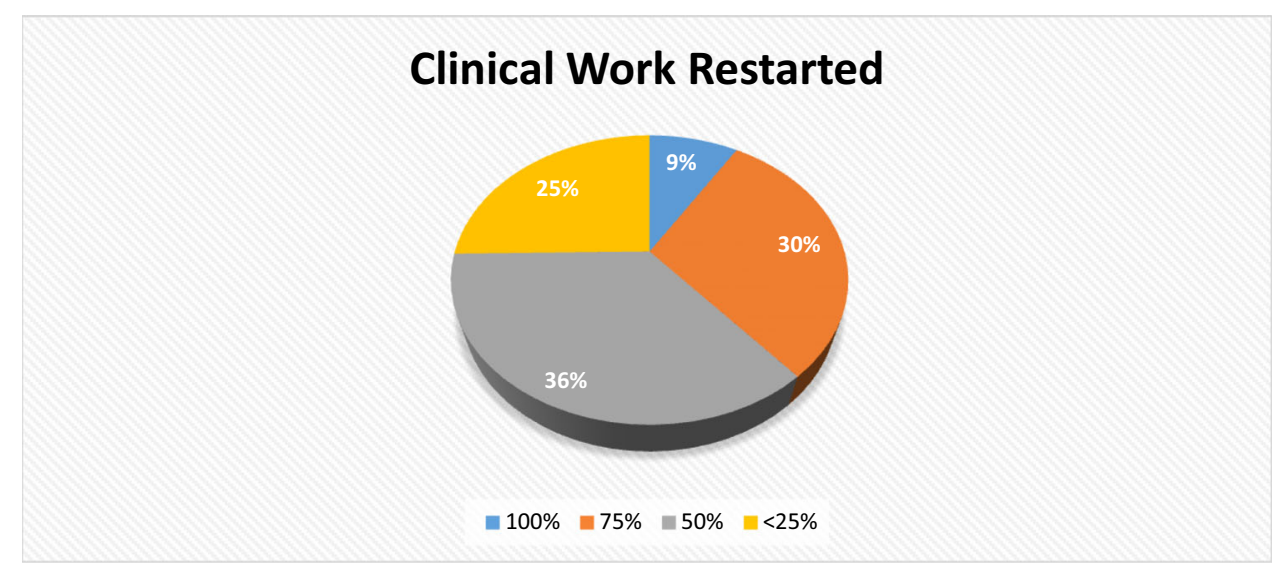

has lesser aerosol producing procedures. Our maximum aerosol generation is during bone cutting.

Though COVID era modifications recommended use of chisel mallet technique or lingual split technique for the same whenever possible. But lingual split till now was not a routine practice as most of our training centres for last few decades were emphasizing on motorized bone removal. This study also reported similar finding as only $2.9 \%$ used chisel mallet and $4.9 \%$ did lingual split technique when work was reinstated. May be this catalyses us to train our students in conventional techniques too. Physiodispensor at low speed and torque is another mechanism advocated to minimise aerosol production during bone removal, but only $8.7 \%$ respondents claim its use during and after work reinstated during COVID pandemic(Graph 2).

COVID-19 pandemic has affected, but in a way revolutionized all levels of the education system (9). Healthrelated educational institutions did assume a partial closure to physical academic activities, but partial clinical teaching on minimum work force still continued to some extent. This extra available time enabled an online mode of scientific information dissemination globally and regular postgraduate academic activities too were conducted via various suddenly popularized portals (Graph 3). 51.5\% respondents believed that there was an escalation in conductance of postgraduate academic sessions as well while $15 \%$ reported it was unchanged. $80.6 \%$ reported that online mode of teaching was primarily used despite when physical clinical work was started on gradient mode (Graph 4). However only $25 \%$ were satisfied and believed that these online activities can replicate seminar room experience (Table 1).

The discrepancy between online and offline seminar performance can be attributed to technical glitches with still refining platforms, internet connections or may be lack of possibility of ensuring the decorum (Table 1) addressal of these aspects may make online teaching more conducive and worthy [4].

In India, most of postgraduate teaching is in form of seminars, journal clubs or case discussions by postgraduates themselves. Regular formal lectures or sessions by faculty are hardly conducted. But there was a plethora of webinars by different centres in which teachers especially senior faculties were speakers or moderators. $90 \%$ appreciated this privilege of listening to their own faculties and ones outside their centres at convenience of a click. Almost all of them liked the opportunity bestowed by COVID to listen to their own faculty even if in PAN India webinars (Table 1). These students advocate continual of such 
Graph 2 Use of different techniques for bone cutting

Graph 3 Use of different methods of teaching mode during COVID era

\section{Method Of Bone Cutting}

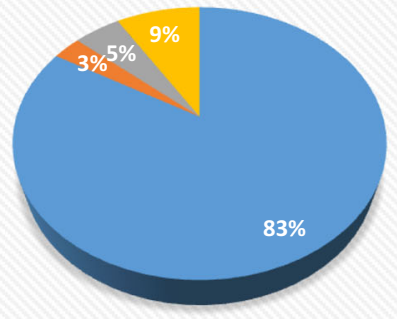

Micromotor $\quad$ Chisel and mallet on buccal side $\quad$ Lingual split technique $\quad$ Physiodispenser

\section{Departmental Academic Activities}

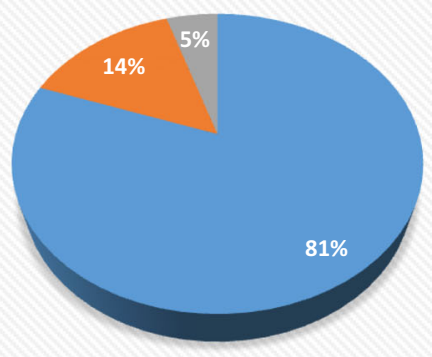

=ONLINE $=$ OFFLINE $\because$ SUSPENDED/NOT CONDUCTED

\section{TRENDS OF ACADEMIC ACTIVITIES}

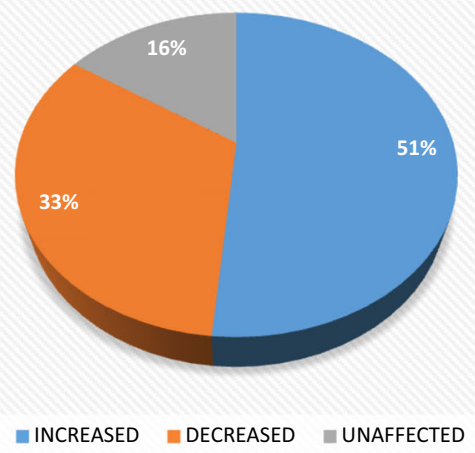

few had to be chosen. But continuous overflow of webinars eventually reduced the enthusiasm of attending them as reported by $28.1 \%$ of postgraduates. $87 \%$ believed excessive long duration of webinars was also a cause for diminution of attention span and enthusiasm (Table 1).

COVID has brought about immense and even unimaginable changes in our lives. It must be the first time in history that postgraduate examination of clinical specialities were conducted on virtual modes. More than $60 \%$ of $75 \%$ felt that a phase came when these webinars were so huge in number that it was difficult to attend them all and 
students themselves felt that online examination was not satisfactory to assess any candidate. How well the extent of skill evaluation can be done by virtual cases or scenarios is a matter of debate. But surely it ventures us to mend ways to substantiate this online mode of evaluation by indulging more realism to the examination pattern.

97\% of respondents felt that this pandemic did affect their professional training considerably such that both clinical exposure and academics were affected (Table 1). Unexpectedly $56.3 \%$ of them even accepted a suggestion of extension of their programme by an additional period to cover up the learning gaps. This clearly highlights the insecurities in their minds regarding learning deficit. No doubt this pandemic had a worse hit on many aspects but at the same time has been an experience of lifetime. Constraints lead to opportunities and a need drives an invention. May be this consummate global pandemic has paved and raced the due incorporation of technology into medical education. Medical education however cannot be done on purely virtual ways with the existing technological support. This has further engraved the need for human replacement of "Feel of touch" by robotics and haptics for the times of crisis. If and when introduced, this will also open the avenues for distant real-time trainings and shrink the world to the size of a palm held device [2].

\section{Conclusion}

The survey highlighted the intense negative impacts of this pandemic on the postgraduate programme from the eyes of the trainees themselves. Last few years had shown an attempt for revolution of medical teaching with unconventional learning methods, case and scenario-based learnings. This triggers us to hasten this process of medical education transformation in a way to cope with any such calamity with minimal consequences.

Funding No funding was received for conduction of this study.

\section{Declarations}

Conflicts of interest The authors declare that they have no conflict of interest.

\section{References}

1. Mahdy MAA (2020) The impact of COVID-19 pandemic on the academic performance of veterinary medical students. Front Vet Sci 7:1-8

2. Alsoufi A, Alsuyihili A, Msherghi A, Elhadi A, Atiyah H, Ashini A et al (2020) Impact of the COVID-19 pandemic on medical education: medical students' knowledge, attitudes, and practices regarding electronic learning. PLoS ONE 15:1-20

3. Rose S (2020) Medical student education in the time of COVID19. JAMA 323(21):2131-2132

4. Hilburg R, Patel N, Ambruso S, Biewald MA, Farouk SS (2020) Medical education during the coronavirus disease-2019 pandemic: learning from a distance. Adv Chronic Kidney Dis 27(5):412-417

Publisher's Note Springer Nature remains neutral with regard to jurisdictional claims in published maps and institutional affiliations. 